\title{
A phase 1 study of dalpiciclib, a cyclin- dependent kinase 4/6 inhibitor in Chinese patients with advanced breast cancer
}

Pin Zhang ${ }^{1}$, Binghe $\mathrm{Xu}^{{ }^{*}}{ }^{*}$, Lin Gui ${ }^{1}$, Wenna Wang ${ }^{1}$, Meng Xiu ${ }^{1}$, Xiao Zhang ${ }^{2}$, Guilan Sun ${ }^{2}$, Xiaoyu Zhu ${ }^{2}$ and Jianjun Zou ${ }^{2}$

\begin{abstract}
Background: Dalpiciclib (SHR6390) is a novel inhibitor of cyclin-dependent kinase 4/6 which demonstrated promising anti-tumor potency in preclinical models. This first-in-human study was conducted to evaluate the tolerability, pharmacokinetics, safety, and preliminary antitumor activity of dalpiciclib in patients with advanced breast cancer $(A B C)$.
\end{abstract}

Methods: In this open-label, phase 1 study, Chinese patients who had failed standard therapy were enrolled to receive oral dalpiciclib in 3+3 dose-escalation pattern at doses of 25-175 mg. Eligible patients were given a singledose of dalpiciclib in week 1, followed by once daily continuous doses for 3 weeks, and 1 week off in 28-day cycles. Based on the tolerability, pharmacokinetics, and activity data revealed from the dose-escalation phase, three dose cohorts were selected to expand to 8-10 patients. The primary endpoints were maximum tolerated dose (MTD) and pharmacokinetics.

Results: Between Apr 15, 2016 and Dec 21, 2018, 40 patients were enrolled; all were diagnosed of hormone receptor-positive and HER2-negative ABC. Dalpiciclib $100 \mathrm{mg}, 125 \mathrm{mg}$, and $150 \mathrm{mg}$ cohorts were expanded to 10 patients. No dose-limiting toxicity was observed and the MTD was not reached. Adverse events (AEs) of grade 3 or 4 were observed in $22(55.0 \%)$ of 40 patients, being neutropenia (52.5\%), leukopenia (35.0\%), thrombocytopenia (5.0\%), and hypertension (2.5\%). No serious AEs were reported. At the doses of 50-175 mg, steady state areas under the concentration-time curve and peak concentration increased almost proportionally with dose. The disease control rate (DCR) was 62.5\% (25/40, 95\% Cl: 45.8-77.3). Two patients (5\%; $125 \mathrm{mg}$ and $150 \mathrm{mg}$ cohorts) achieved partial response, with responses lasting 169 and 356+ days, respectively. Among the three expansion cohorts, the $150 \mathrm{mg}$ cohort had the numerically highest DCR of $80.0 \%$ (95\% Cl: 44.4-97.5) and longest median progression-free survival of 8.4 months (95\% Cl: 2.1 -not reached).

Conclusions: Dalpiciclib showed acceptable safety profile and dose-dependent plasma exposure in Chinese patients with ABC. The recommended phase 2 dose was $150 \mathrm{mg}$. Preliminary evidence of clinical activity was observed, which warrants further validation.

(Continued on next page)

\footnotetext{
* Correspondence: bhxu@hotmail.com

1 National Cancer Center/Cancer Hospital, Chinese Academy of Medical

Sciences and Peking Union Medical College, Beijing, China

Full list of author information is available at the end of the article
}

\section{$\triangle B M C$}

C C The Author(s). 2021 Open Access This article is licensed under a Creative Commons Attribution 4.0 International License, which permits use, sharing, adaptation, distribution and reproduction in any medium or format, as long as you give appropriate credit to the original author(s) and the source, provide a link to the Creative Commons licence, and indicate if changes were made. The images or other third party material in this article are included in the article's Creative Commons licence, unless indicated otherwise in a credit line to the material. If material is not included in the article's Creative Commons licence and your intended use is not permitted by statutory regulation or exceeds the permitted use, you will need to obtain permission directly from the copyright holder. To view a copy of this licence, visit http://creativecommons.org/licenses/by/4.0/. The Creative Commons Public Domain Dedication waiver (http://creativecommons.org/publicdomain/zero/1.0/) applies to the data made available in this article, unless otherwise stated in a credit line to the data. 
(Continued from previous page)

Trial registration: ClinicalTrials.gov identifier: NCT02684266. Registered Feb 17, 2016.

Keywords: Cyclin-dependent kinase 4/6 inhibitor, Advanced breast cancer, First-in-human trial

\section{Background}

Cell cycle dysregulation and aberrant cell proliferation are a hallmark of cancer [1]. The cyclin D (CCND)-cyclindependent kinase 4/6 axis (CDK4/6), which modulates the transition through the $G_{1}$ phase to $S$ phase of the cell cycle, plays a key role in the pathological process of many cancer types [2]. CDK 4 and 6 can interact with CCNDs to promote the phosphorylation of the tumor-suppressor retinoblastoma protein $(\mathrm{Rb})$ and the release of $\mathrm{Rb}$-bound E2F transcription factor, enabling cell cycle progression from $G_{1}$ [3]. In addition, selective CDK4/6 inhibition allows preferential inhibition of oncogenic events while sparing toxicity in normal tissues and therefore represents an appealing therapeutic strategy for cancer $[3,4]$.

Endocrine therapy is the cornerstone in the treatment of hormone receptor (HR)-positive advanced breast cancer $(A B C)$. Nevertheless, acquired resistance to endocrine therapy inevitably develops during the course of treatment [5]. Estrogen-mediated hyperactivity of the CCND-CDK4/6 axis is a central feature of HR-positive breast cancer, and the tumors usually retain a functional $\mathrm{Rb}$ [6], which can be targeted by CDK4/6 inhibitors. To date, three CDK4/6 inhibitors including palbociclib, ribociclib, and abemaciclib have been approved by the US Food and Drug Administration and the European Medicines Agency in combination with endocrine therapy as the first- and second-line treatment for HRpositive and human epidermal growth factor receptor 2 (HER2)-negative $A B C$. In pivotal trials, the addition of these CDK4/6 inhibitors to standard endocrine therapy substantially improved progression-free survival (PFS) and overall survival [7-15]. Abemaciclib has also been approved as a monotherapy for HR-positive and HER2negative breast cancer progressing on prior endocrine therapy or chemotherapy in the metastatic setting [16].

Dalpiciclib (SHR6390) is a novel, highly selective, small molecule CDK4/6 inhibitor with comparable potencies against CDK4 ( $\left.\mathrm{IC}_{50}, 12.4 \mathrm{nM}\right)$ and CDK6 ( $\left.\mathrm{IC}_{50}, 9.9 \mathrm{nM}\right)$. Dalpiciclib has demonstrated anti-tumor activity in a variety of in vitro and xenograft models primarily via Rb-dependent cytostasis [17, 18]. In vivo xenografts, dalpiciclib generally showed similar or slightly better anti-tumor potency compared with palbociclib, without inducing noticeable toxicity [18]. Moreover, in HRpositive breast cancer cell lines and xenografts, dalpiciclib could overcome the acquired drug resistance to endocrine therapy [18]. Based on these preclinical evidence, we conducted a first-in-human, phase 1 trial to assess the safety, tolerability, pharmacokinetics (PK) and preliminary efficacy of oral dalpiciclib in patients with HR-positive and HER2-negative ABC.

\section{Methods \\ Study design}

This open-label, phase 1 trial in patients with $A B C$ was conducted in China (ClinicalTrials.gov identifier: NCT02684266). The study consisted of dose-escalation and dose-expansion phases. In the dose-escalation phase, the primary objective was to establish the maximum tolerated dose (MTD) for oral dalpiciclib. In the doseexpansion phase, three selected dose cohorts were expanded to further characterize the safety profile, tolerability, PK parameters and preliminary anti-tumor activity of dalpiciclib (Fig. 1).

\section{Patients}

Eligible patients were aged 18-65 years, with histologically confirmed $\mathrm{ABC}$ who failed standard therapy. Other inclusion criteria included Eastern Cooperative Oncology Group performance status 0-1, life expectancy $\geq 3$ months, adequate bone marrow function (hemoglobin $>110 \mathrm{~g} / \mathrm{L}$, neutrophils $>2.0 \times 10^{9}$ per $\mathrm{L}$ and platelets > $100 \times 10^{9}$ per L), adequate liver and renal function (total bilirubin $\leq 1.5$ times the upper limit of normal [ULN], alanine aminotransferase [ALT] and aspartate aminotransferase $[\mathrm{AST}] \leq 1.5$ times ULN $[\leq 5$ times ULN in the presence of liver metastases] and creatinine $\leq 1$ ULN), and adequate cardiac function (left ventricular ejection fraction $\geq 50 \%$ and Fridericia corrected-QT [QTcF] interval $<$ $450 \mathrm{~ms}$ in males or $<470 \mathrm{~ms}$ in females). The key exclusion criteria were prior or current treatment with CDK4/6 targeted therapy, cytotoxic chemotherapy within 3 weeks (6 weeks for mitomycin $\mathrm{C}$ or nitrosamine) or any other anti-tumor therapy within 3 weeks (except for endocrine therapy, which had to be discontinued before the time of informed consent), untreated or uncontrolled/unstable brain metastases (as judged by the investigator) or requirement of long-term use of steroids.

\section{Procedures}

For dose escalation, a traditional $3+3$ design was used with three to six patients enrolled per dose level and the escalation continued until two or more patients had dose-limiting toxicities (DLTs) in one dose cohort during the DLT assessment period (from the administration of the first study dose to the end of the first cycle). The 


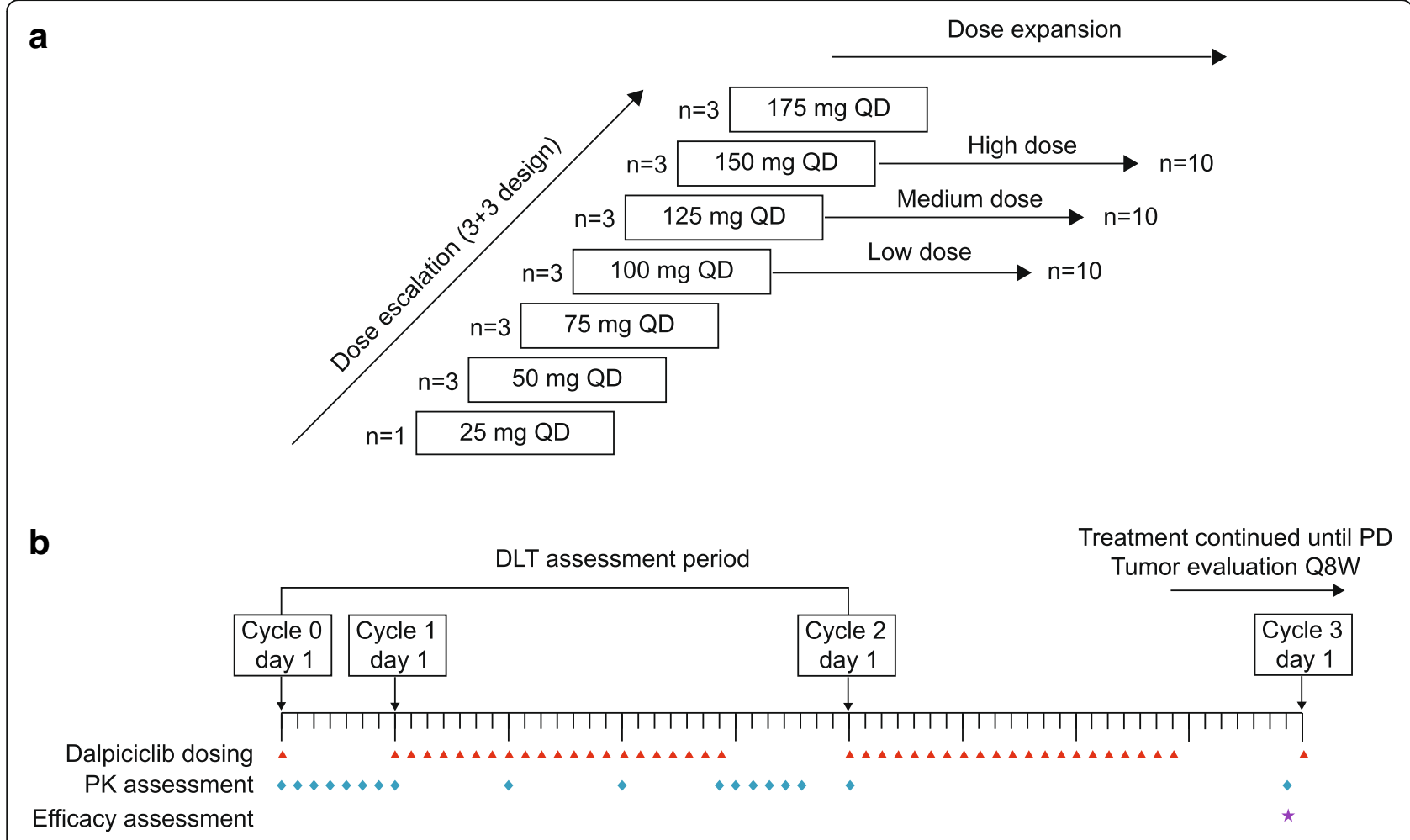

Fig. 1 Study design. a Framework for the dose-escalation and dose-expansion study of dalpiciclib. b Dosing and assessment schema. DLT, doselimiting toxicity; PD, progressive disease

starting dose of dalpiciclib was $25 \mathrm{mg}$ and was escalated by $25 \mathrm{mg}$ increments up to $175 \mathrm{mg}(25 \mathrm{mg}, 50 \mathrm{mg}, 75$ $\mathrm{mg}, 100 \mathrm{mg}, 125 \mathrm{mg}, 150 \mathrm{mg}$ and $175 \mathrm{mg}$ ) in a modified Fibonacci schema (Fig. 1a). At the $25 \mathrm{mg}$ dose level, one patient was enrolled as a sentinel; if no grade $\geq 2$ toxicity was observed during the sentinel patient's DLT assessment period, escalation could proceed to the $50 \mathrm{mg}$ dose level immediately. DLT was defined as an adverse event (AE) that met any of the following criteria: grade 4 hematologic toxicity, grade 3 neutropenia with infection or fever $\geq 38.5^{\circ} \mathrm{C}$, grade 3 thrombocytopenia with apparent clinical bleeding tendency, grade $\geq 3$ nonhematologic toxicity (excluding untreated nausea, vomiting, and diarrhea or AEs considered tolerable by the patients, such as alopecia) or grade $\geq 2$ cardiac or renal toxicity. Based on the tolerability, PK, and anti-tumor activity revealed from the dose-escalation phase, three dose cohorts were selected to expand to 8-10 patients.

All patients were first given a single dose of oral dalpiciclib. After $a \geq 7$-day washout period, the patient was then administered once-daily continuous doses of dalpiciclib for 3 weeks in 28-day cycles (Fig. 1b). Dose interruption or reduction was permitted after the initiation of the second cycle of treatment as prespecified in protocol (Additional file 1: Table S1). Patients who achieved complete response $[\mathrm{CR}]$, partial response $[\mathrm{PR}]$ or stable disease [SD]) at first efficacy assessment after receiving two cycles of dalpiciclib could continue treatment until disease progression, intolerable toxicity, withdrawal of patient consent or investigator decision.

\section{Endpoint}

The primary endpoint was the MTD of dalpiciclib and pharmacokinetic parameters in patients with $\mathrm{ABC}$. The MTD was the highest dose level at which $<33 \%$ of the patients reported a DLT within the first cycle of multiple dosing. The secondary endpoints included safety and the investigator-assessed objective response rate (ORR, defined as the proportion of patients with CR and PR as the best overall response) and disease control rate (DCR, defined as the proportion of patients with $\mathrm{CR}, \mathrm{PR}$ or $\mathrm{SD} \geq 6$ weeks) per RECIST v1.1.

\section{Assessment}

Safety was regularly monitored from the time of informed consent until 30 days after the last dose of study drug. AEs were graded according to the Common Terminology Criteria for Adverse Events v4.0. Tumor response was assessed using computerized tomography or magnetic resonance imaging at baseline and every two cycles after the start of multiple once-daily dosing. The response was classified as $\mathrm{CR}, \mathrm{PR}, \mathrm{SD}$ or progressive 
disease (PD) according to RECIST v1.1 by the investigators [19]. A CR or PR was required to be confirmed with a subsequent scan 4 weeks after the initial assessment.

For PK analysis, blood samples were collected in all enrolled patients from predose and at intervals up to 24 h $(0.5 \mathrm{~h}, 1 \mathrm{~h}, 2 \mathrm{~h}, 3 \mathrm{~h}, 4 \mathrm{~h}, 6 \mathrm{~h}, 8 \mathrm{~h}, 10 \mathrm{~h}$ and $24 \mathrm{~h})$ on day 1 and every $24 \mathrm{~h}$ thereafter through day 6 at the singledose stage; and on day 1 (predose), day 8 (predose), day 15 (predose), day 21 (predose and $0.5 \mathrm{~h}, 1 \mathrm{~h}, 2 \mathrm{~h}, 3 \mathrm{~h}, 4 \mathrm{~h}$, $6 \mathrm{~h}, 8 \mathrm{~h}$ and $10 \mathrm{~h}$ postdose) and day 22 through day 26 (every $24 \mathrm{~h}$ ) of cycle 1 at the multiple-dose stage. The plasma concentrations of dalpiciclib were measured by liquid chromatography with tandem mass spectrometry.

\section{Statistics}

The DLT analysis set included all patients who received at least one dose of the study drug and completed the first cycle of treatment or discontinued study drug due to $\mathrm{AE}$ during the first cycle of treatment. PK was analyzed in patients who received at least one dose of the study drug and had evaluable post-treatment PK data. Safety and efficacy were analyzed in all patients who received at least one dose of the study drug. Descriptive statistics were reported for the safety and efficacy outcomes and PK parameters. The ORR and DCR were calculated, with the corresponding 95\% Clopper-Pearson confidence intervals (CIs) provided. The Kaplan-Meier method was used to analyze PFS, and the median PFS (month) was estimated. The two-sided 95\% CI of the median PFS was calculated by the Brookmeyer Crowley method. PK parameters were analyzed using the noncompartmental model with WinNonlin 7.0 (Pharsight, Mountain View, CA). Statistical analyses were conducted with SAS 9.4 (SAS Institute Inc., Cary, NC).

\section{Results}

\section{Patient characteristics and deposition}

Between Apr 15, 2016 and Dec 21, 2018, 58 patients were screened. Among them, 40 patients were enrolled and received dalpiciclib. All patients were diagnosed of HR-positive and HER2-negative stage IV ABC. $45.0 \%$ of the patients had at least three lines of prior chemotherapies and $55.0 \%$ had at least two prior endocrine therapies (Table 1). The median follow-up was 7.0 months (range, 2.7-35.0). The main reason for treatment discontinuation was disease progression (28 patients, $70.0 \%$ ). At data cutoff (Jun 28, 2019), treatment was still on-going in 10 patients (25.0\%). No deaths were reported.

Table 1 Demographics and baseline disease characteristics

\begin{tabular}{|c|c|c|c|c|c|c|c|c|}
\hline & $\begin{array}{l}25 \mathrm{mg} \\
(n=1)\end{array}$ & $\begin{array}{l}50 \mathrm{mg} \\
(n=3)\end{array}$ & $\begin{array}{l}75 \mathrm{mg} \\
(n=3)\end{array}$ & $\begin{array}{l}100 \mathrm{mg} \\
(n=10)\end{array}$ & $\begin{array}{l}125 \mathrm{mg} \\
(n=10)\end{array}$ & $\begin{array}{l}150 \mathrm{mg} \\
(n=10)\end{array}$ & $\begin{array}{l}175 \mathrm{mg} \\
(n=3)\end{array}$ & $\begin{array}{l}\text { All patients } \\
(n=40)\end{array}$ \\
\hline Age, years & $50(50-50)$ & $52(51-64)$ & $53(48-63)$ & $58(39-65)$ & $54(26-63)$ & $55(39-65)$ & $51(51-64)$ & $54(26-65)$ \\
\hline \multicolumn{9}{|l|}{ Sex } \\
\hline Female & $1(100)$ & $3(100)$ & $3(100)$ & $10(100)$ & $10(100)$ & $10(100)$ & $3(100)$ & $40(100)$ \\
\hline \multicolumn{9}{|l|}{$\begin{array}{l}\text { ECOG performance } \\
\text { status }\end{array}$} \\
\hline 0 & $1(100)$ & $3(100)$ & $2(66.7)$ & $4(40.0)$ & $10(100)$ & $9(90.0)$ & $3(100)$ & $32(80.0)$ \\
\hline 1 & 0 & 0 & $1(33.3)$ & $6(60.0)$ & 0 & $1(10.0)$ & 0 & $8(20.0)$ \\
\hline $\begin{array}{l}\text { Time since first } \\
\text { diagnosis, years }\end{array}$ & $10.9(10.9-10.9)$ & $5.2(1.7-11.9)$ & $5.9(2.4-12.0)$ & $9.6(4.9-20.3)$ & $6.8(1.4-20.1)$ & $11.3(1.0-19.6)$ & $8.2(8.2-10.5)$ & $8.8(1.0-20.3)$ \\
\hline \multicolumn{9}{|l|}{$\begin{array}{l}\text { Tumor stage at study } \\
\text { entry }\end{array}$} \\
\hline IV & $1(100)$ & $3(100)$ & $3(100)$ & $10(100)$ & $10(100)$ & $10(100)$ & $3(100)$ & $40(100)$ \\
\hline \multicolumn{9}{|l|}{ Previous therapy } \\
\hline Surgery & $1(100)$ & $3(100)$ & $3(100)$ & $10(100)$ & $10(100)$ & $10(100)$ & $3(100)$ & $40(100)$ \\
\hline Radiotherapy & $1(100)$ & $3(100)$ & $3(100)$ & $8(80.0)$ & $5(50.0)$ & $8(80.0)$ & $3(100)$ & $31(77.5)$ \\
\hline \multicolumn{9}{|l|}{ Chemotherapy } \\
\hline $1-2$ regimens & 0 & 0 & $2(66.7)$ & $2(20.0)$ & $4(40.0)$ & $3(30.0)$ & $2(66.7)$ & $13(32.5)$ \\
\hline 3-6 regimens & $1(100)$ & 0 & $1(33.3)$ & $6(60.0)$ & $4(40.0)$ & $5(50.0)$ & $1(33.3)$ & $18(45.0)$ \\
\hline \multicolumn{9}{|l|}{ Endocrine therapy } \\
\hline $1-2$ regimens & 0 & $1(33.3)$ & $2(66.7)$ & $4(40.0)$ & $4(40.0)$ & $6(60.0)$ & $3(100)$ & $20(50.0)$ \\
\hline 3-6 regimens & $1(100)$ & 0 & $1(33.3)$ & $3(30.0)$ & $3(30.0)$ & $3(30.0)$ & 0 & $11(27.5)$ \\
\hline
\end{tabular}

Data are $\mathrm{n}(\%)$ or median (range) 


\section{Safety and tolerability}

Dalpiciclib was dose escalated from $25 \mathrm{mg}$ QD to 175 mg QD. No DLT was observed in all dose cohorts within the first cycle of treatment and the MTD was not reached. With $\mathrm{PK}$ analysis showing increased exposure $\left(C_{\max }, C_{\min }, A C_{s s}\right)$ of dalpiciclib and increased occurrence of neutropenia and leukopenia (indicator of pharmacodynamics) within first cycle at doses of 50$150 \mathrm{mg}$, and the best overall response of SD or better for all patients at $100-150 \mathrm{mg}(4 / 7 \mathrm{PD}$ at $25-75 \mathrm{mg}$ and $1 / 3$ PD at $175 \mathrm{mg}$ ), $150 \mathrm{mg}$ was first selected for expansion. Then with safety as a main consideration $(8 / 10$ with grade 3 and $1 / 10$ with grade 4 neutropenia for the 150 mg cohort), the $125 \mathrm{mg}$ and $100 \mathrm{mg}$ cohorts were subsequently expanded to 10 patients. Overall, no DLT was observed in the expansion cohorts within the prespecified assessment window.

The median duration of treatment exposure was 123 days (range, 56-1063). No patients discontinued treatment due to AEs. All patients in the study had at least one treatment-emergent AE (TEAE). Common TEAEs and all TEAEs occurring in $\geq 2$ patients are provided in Table 2 and Additional file 1 Table S2 respectively. The most frequent non-hematological toxicities were increased AST (40.0\%) and ALT (32.5\%); of these, 2 cases of increased AST and 1 case of increased ALT were of grade 2 severity and all others were of grade 1 . QT prolongation was reported for 5 (12.5\%) patients, including 3 with a maximum QTcF interval of $<480 \mathrm{~ms}$ (grade 1 ) and 2 with a maximum interval of $480-500 \mathrm{~ms}$ (grade
2). No patient had a maximum value of $>500 \mathrm{~ms}$ or a $>$ $60 \mathrm{~ms}$ increase from baseline. Among the 5 cases with QT prolongation, 2 (5\%; both grade 1) were considered possibly treatment-related by the investigators. Thrombosis (grade 1) was reported in 1 patient and judged as treatment unrelated. TEAEs of grade 3 or 4 were observed in $22(55.0 \%)$ of 40 patients, being neutropenia (52.5\%), leukopenia (35.0\%), thrombocytopenia (5.0\%), and hypertension (2.5\%). No serious AEs or grade $5 \mathrm{AEs}$ were reported.

PK

PK parameters following single and multiple dosing of dalpiciclib are presented in Table 3 and the plasma concentration-time curves are shown in Additional file 1 Figure S1. At doses of $50-175 \mathrm{mg}$, the median time to peak concentration was $2.5-4.0 \mathrm{~h}$, and the geometric mean terminal half-life was $40.3-51.4 \mathrm{~h}$ after a single dose of dalpiciclib. Following multiple dosing, steady state dalpiciclib was observed on day 8 . The median time to peak concentration was $3.0-4.0 \mathrm{~h}$, and the geometric mean terminal half-life was $44.9-52.3 \mathrm{~h}$ at steady state on day 21 and the mean accumulation ratio for area under the concentration-time curve was 1.9-3.4 across the doses of $50-175 \mathrm{mg}$. The area under the concentration-time curve at steady state and peak concentration increased almost proportionally with dose increase over the dosing range of $50-175 \mathrm{mg}$. The steady state $C_{\max }$ at day 21 was 41.1, 53.4, 87.0, 115, 126, and

Table 2 Treatment-emergent adverse events (TEAEs) occurring in $\geq 20 \%$ of patients

\begin{tabular}{|c|c|c|c|}
\hline & \multicolumn{3}{|c|}{ All patients $(n=40)$} \\
\hline & All Grades & Grade 3 & Grade 4 \\
\hline \multicolumn{4}{|l|}{ Hematologic TEAEs } \\
\hline Neutropenia & $40(100)$ & $19(47.5)$ & $2(5.0)$ \\
\hline Leukopenia & $40(100)$ & $13(32.5)$ & $1(2.5)$ \\
\hline Anemia & $13(32.5)$ & 0 & 0 \\
\hline Thrombocytopenia & $11(27.5)$ & $1(2.5)$ & $1(2.5)$ \\
\hline \multicolumn{4}{|l|}{ Non-hematologic TEAEs } \\
\hline Aspartate aminotransferase increased & $16(40.0)$ & 0 & 0 \\
\hline Fatigue & $15(37.5)$ & 0 & 0 \\
\hline Blood creatinine increased & $14(35.0)$ & 0 & 0 \\
\hline Alanine aminotransferase increased & $13(32.5)$ & 0 & 0 \\
\hline Headache & $13(32.5)$ & 0 & 0 \\
\hline Alopecia & $11(27.5)$ & 0 & 0 \\
\hline Bilirubin conjugated increased & $10(25.0)$ & 0 & 0 \\
\hline Decreased appetite & $9(22.5)$ & 0 & 0 \\
\hline Constipation & $8(20.0)$ & 0 & 0 \\
\hline Dyspnoea & $8(20.0)$ & 0 & 0 \\
\hline
\end{tabular}

Data are $\mathrm{n}(\%)$. No grade 5 adverse events occurred 
Table 3 Plasma pharmacokinetics of single and multiple dosing of dalpiciclib

\begin{tabular}{|c|c|c|c|c|c|c|}
\hline & $50 \mathrm{mg}$ QD & $75 \mathrm{mg}$ QD & $100 \mathrm{mg}$ QD & $125 \mathrm{mg}$ QD & $150 \mathrm{mg}$ QD & $175 \mathrm{mg} \mathrm{QD}$ \\
\hline Single dosing & $n=3$ & $n=3$ & $n=10$ & $n=10$ & $n=10$ & $n=3$ \\
\hline$T_{\max ,} h$ & $3.0(2.0-4.0)$ & $3.0(3.0-3.0)$ & $2.5(2.0-4.0)$ & $3.5(2.0-6.0)$ & $3.5(2.0-4.0)$ & $4.0(3.0-4.0)$ \\
\hline$C_{\max }, \mathrm{ng} / \mathrm{mL}$ & $12.2(57.3)$ & $20.3(37.3)$ & $46.8(27.5)$ & $46.6(56.8)$ & $63.6(64.4)$ & $114(37.8)$ \\
\hline$A \cup C_{0-t}, h^{*} n g / m L$ & $482(40)$ & $572(13)$ & $1170(20)$ & $1480(38)$ & $1880(57)$ & $3080(24)$ \\
\hline$t_{1 / 2 z}, h$ & $51.0(13.6)$ & $46.9(8.0)$ & 49.7 (16.6) & 43.7 (23.5) & $51.4(23.2)$ & $40.3(22.4)$ \\
\hline$C L / F, L / h$ & 89.8 (39.2) & $117(15.7)$ & 76.1 (20.7) & 76.6 (37.8) & 70.5 (51.9) & $52.8(21.8)$ \\
\hline$V_{z} / F, L$ & $6610(40)$ & 7900 (12) & $5450(25)$ & $4830(45)$ & $5230(71)$ & 3080 (39) \\
\hline Multiple dosing & $n=3$ & $n=3$ & $n=9$ & $n=9$ & $n=9$ & $n=3$ \\
\hline$T_{\max }, h$ & $4.0(4.0-6.0)$ & $3.0(3.0-4.0)$ & $3.0(2.0-6.0)$ & $4.0(2.0-6.0)$ & $4.0(2.0-8.0)$ & $3.0(3.0-3.0)$ \\
\hline$C_{\max }, n g / m L$ & $41.1(23.9)$ & $53.4(9.4)$ & $87.0(48.5)$ & 115 (28.7) & $126(30.0)$ & 155 (57.8) \\
\hline $\mathrm{AUC}_{5 \mathrm{~s}}, \mathrm{~h}^{*} \mathrm{ng} / \mathrm{mL}$ & $615(27)$ & 792 (18) & $1410(53)$ & 2020 (32) & $2230(24)$ & $2730(48)$ \\
\hline$t_{1 / 2 z}, h$ & 46.9 (11.9) & $52.3(6.3)$ & $48.2(20.3)$ & $44.9(21.2)$ & $44.9(17.5)$ & $45.1(6.6)$ \\
\hline $\mathrm{CL}_{s s} / \mathrm{F}, \mathrm{L} / \mathrm{h}$ & $81.4(26.9)$ & 94.7 (18.0) & $71.0(52.5)$ & $62.0(31.8)$ & $67.2(23.9)$ & $64.1(47.8)$ \\
\hline$V_{z} / F, L$ & 5510 & $6920(31)$ & $4930(67)$ & $4010(30)$ & $4350(32)$ & $4170(48)$ \\
\hline$R_{a c c(A \cup C)}$ & $3.4(41.3)$ & $3.0(22.1)$ & $2.7(35.9)$ & $3.3(60.0)$ & $2.7(52.8)$ & $1.9(37.7)$ \\
\hline
\end{tabular}

Data are median (range) for $\mathrm{T}_{\max }$ and geometric mean (geometric coefficient of variation\%) for others

$T_{\max }$ time to reach $C_{\max }, C_{\max }$ peak plasma concentration, $A \cup C_{0-t}$ area under the curve from time 0 to the last measurable concentration, $A U C_{s s}$ area under the curve for dose interval, $t_{1 / 2}$ terminal half-life, $C L / F$ apparent clearance, $V_{z} / F$ apparent volume of distribution, $R_{a c}(A \cup C$ ) accumulation ratio for AUC

$155 \mathrm{ng} / \mathrm{mL}$ in the $50,75,100,125,150$, and $175 \mathrm{mg}$ cohorts, respectively (Table 3).

\section{Efficacy}

All 40 patients with HR-positive and HER2-negative $\mathrm{ABC}$ were evaluable for tumor response. Of those, two patients (5\%) achieved PR: one patient (125 mg cohort) had previously received two lines of chemotherapy for $\mathrm{ABC}$ and partial response was initially observed at cycle8 tumor assessment visit and lasted for 169 days; the other patient ( $150 \mathrm{mg}$ cohort) had previously received six regimens of endocrine therapy and partial response was initially documented at cycle- 4 tumor assessment visit and lasted for 356+ days. A total of 23 (57.5\%) patients across $50-175 \mathrm{mg}$ dose cohorts had best overall response with stable disease and the median duration was 4.0 months (inter-quartile range, 2.1-7.6). The overall DCR was $62.5 \%$ (95\% CI: 45.8-77.3) in all patients (Table 4). By data cutoff, a total of 26 disease progression events occurred. Median PFS was 4.0 months (95\% CI: 2.4-9.1) in all 40 patients and 5.9 months (95\% CI: 2.4-9.1) in 30 patients at the three dose levels with expansion cohorts. Among the three dose cohorts with expansion, the $150 \mathrm{mg}$ cohort had the numerically highest DCR of $80.0 \%$ (95\% CI: 44.4-97.5) and longest median PFS of 8.4 months (95\% CI: 2.1-not reached).

\section{Discussion}

The findings from this first-in-human, phase 1 trial showed that oral dalpiciclib with a 3-week on and 1week off dosing regimen was well-tolerated in patients with $\mathrm{ABC}$, with no MTD observed up to the highest dose tested. TEAEs were generally manageable with no treatment discontinuation due to AEs or serious AEs documented. At doses of 50-175 mg, plasma exposure increased almost proportionally with dose. Preliminary

Table 4 Tumor response per RECIST v1.1

\begin{tabular}{|c|c|c|c|c|c|c|c|c|}
\hline & $\begin{array}{l}25 \mathrm{mg} \\
(n=1)\end{array}$ & $\begin{array}{l}50 \mathrm{mg} \\
(n=3)\end{array}$ & $\begin{array}{l}75 \mathrm{mg} \\
(n=3)\end{array}$ & $\begin{array}{l}100 \mathrm{mg} \\
(n=10)\end{array}$ & $\begin{array}{l}125 \mathrm{mg} \\
(n=10)\end{array}$ & $\begin{array}{l}150 \mathrm{mg} \\
(n=10)\end{array}$ & $\begin{array}{l}175 \mathrm{mg} \\
(n=3)\end{array}$ & $\begin{array}{l}\text { All patients } \\
(n=40)\end{array}$ \\
\hline \multicolumn{9}{|c|}{ Best overall response, n (\%) } \\
\hline Complete response & 0 & 0 & 0 & 0 & 0 & 0 & 0 & 0 \\
\hline Partial response & 0 & 0 & 0 & 0 & $1(10.0)$ & $1(10.0)$ & 0 & $2(5.0)$ \\
\hline Stable disease & 0 & $2(66.7)$ & $1(33.3)$ & $7(70.0)$ & $4(40.0)$ & $7(70.0)$ & $2(66.7)$ & $23(57.5)$ \\
\hline Progressive disease & 1 & $1(33.3)$ & $2(66.7)$ & $3(30.0)$ & $5(50.0)$ & $2(20.0)$ & $1(33.3)$ & $15(37.5)$ \\
\hline DCR, \% (95\% Cl) & 0 (NR) & $66.7(9.4-99.2)$ & $33.3(0.8-90.6)$ & $70.0(34.8-93.3)$ & $50.0(18.7-81.3)$ & $80.0(44.4-97.5)$ & $66.7(9.4-99.2)$ & $62.5(45.8-77.3)$ \\
\hline
\end{tabular}

$D C R$ disease control rate, NR not reached, RECIST response evaluation criteria in solid tumors 
evidence of clinical activity of dalpiciclib in HR-positive and HER2-negative ABC was also observed, with a DCR of $62.5 \%$.

Dalpiciclib was rapidly absorbed and reached $\mathrm{C}_{\max }$ within 2.5-4.0 h upon intake. No saturation of absorption was observed at doses of 50-175 mg; together with the terminal half-life of $40-51 \mathrm{~h}$, the PK data of dalpiciclib supported the once-daily administration schedule. Dalpiciclib showed a large apparent volume of distribution (3080-7900 L), suggesting that it was widely distributed in the body. In our preclinical tissue distribution study in rats, $\left[{ }^{14} \mathrm{C}\right]$ dalpiciclib was found widely distributed in all tissues with limited brain penetration (unpublished data). In vivo study has showed that dalpiciclib was a P-gp substrate. Since membrane transporters at the blood brain barrier are involved in the regulation of brain penetration of CDK4/6 inhibitors, their interaction with dalpiciclib requires further investigation [20]. Similar to other CDK4/6 inhibitors, dalpiciclib was mainly metabolized in the liver via CYP3A4. In addition, CYP2C9 and CYP2C8 also mediated part of the metabolism of dalpiciclib. Therefore, the exposure of dalpiciclib might be affected by inhibitors or inducers of these enzymes. In vivo drug-drug interaction studies with itraconazole, rifampin and other drugs are underway and the results will provide guidance on clinical concomitant medications. The pharmacokinetic characteristics of dalpiciclib and other approved CDK4/6 inhibitors are provided in detail in Additional file 1 Table S3.

The definitions of DLT and MTD used in our study were accordant with those previously used in studies on palbociclib [21]. During dose-escalation and expansion, no DLT was observed in all patients within the first cycle of treatment. In the phase 1 dose-escalation study of palbociclib, DLT was reported in $5(12 \%)$ patients, all being neutropenia and the MTD and the recommended phase 2 dose was established as $125 \mathrm{mg}$ [21]. In the phase 1 study of ribociclib, DLTs were reported in 9 (13\%) patients within the first treatment cycle, including 3 cases of neutropenia, 2 cases of thrombocytopenia, and 1 each of mucositis, pulmonary embolism, QTcF prolongation and increased creatinine [22]. Consistent with the safety profile of other CDK4/6 inhibitors with similar potencies for CDK4 and CDK6 (palbociclib and ribociclib) [22-24], the most frequent grade 3 or 4 AEs of dalpiciclib were neutropenia and leukopenia, observed in 52.5 and $35.0 \%$ of patients respectively. All the hematologic AEs were managed with dose interruption and/or reduction and standard supportive care (granulocyte colony-stimulating factor etc.). The abnormalities resolved immediately with no related complications such as fever or infection observed. The myelosuppressive effects of dalpiciclib were considered an on-target effect due to the involvement of CDK6 in the proliferation of hematological precursors [25] and the cytostasis induced in $G_{1}$ phase by dalpiciclib was reversible when the drug was held. Abemaciclib shows a different safety profile with higher gastrointestinal toxicity and less hematological toxicity, which might be attributed to its higher potency against CDK4 than CDK6 and additional potency against CDK9 $[9,26,27]$. Of note, the incidence of increased AST and ALT were observed in 40 and $32.5 \%$ of patients respectively in this study, probably due to the high prevalence of liver metastases at baseline $(47.5 \% ; 19 / 40)$ and patient fragility from heavy pretreatment $(45.0 \%$ patients with $\geq 3$ prior lines of chemotherapy, $27.5 \%$ with $\geq 3$ prior lines of endocrine therapy). However, the hepatotoxicity was mild with only 3 cases of grade 2 and no case of grade $\geq 3$ documented. QTcF prolongation was previously reported in $6-9 \%$ patients with ribociclib $[8,22]$; in the phase 1 trial of palbociclib, 26 of 41 patients had a maximum increase from baseline QTc of $<30 \mathrm{~ms}$, but none had a QTc value of $>500 \mathrm{~ms}$ [21]. Prolongation of the QT interval was observed in $12.5 \%$ (all with a maximum value of $<500 \mathrm{~ms}$ ) of patients during treatment with dalpiciclib; nevertheless, only 2 cases were considered treatment-related and both resolved without medical intervention. Lung inflammation, a rare but serious adverse effect reported for other CDK4/6 inhibitors [28], was not observed in the present study, possibly due to the relative small sample size. Taken together, dalpiciclib with a 3-week on/1-week off dosing regimen is well-tolerated with a manageable safety profile in patients with $\mathrm{ABC}$.

Despite the initial success of endocrine therapy in the treatment of HR-positive and HER2-negative ABC, nearly all patients will develop acquired drug resistance with prolonged treatment [5]. In this phase 1 trial, clinical activity of single agent dalpiciclib in HR-positive and HER2-negative $A B C$ was demonstrated with an overall DCR of $62.5 \%$ (95\% CI: 45.8-77.3) across all dose levels, consistent with the preclinical evidence that dalpiciclib could overcome the resistance to an endocrine agent (tamoxifen) [18]. Despite the modest ORR rate (5\%), dalpiciclib was notable for the durable disease control, with a median duration of 4.0 months. Among all therapeutic dose levels with an expansion cohort (100-150 mg), patients treated with $150 \mathrm{mg}$ QD dalpiciclib appeared to derive the greatest clinical benefit, with a DCR of $80.0 \%$ (95\% CI: 44.4-97.5) and a median PFS of 8.4 months. Taken together with the dose-dependent increase in systemic exposure of dalpiciclib $\left(C_{\max }, C_{\min }, A U C_{s s}\right)$ and the overall tolerability at doses of $100-150 \mathrm{mg}, 150 \mathrm{mg}$ QD was selected as the recommended phase 2 dose for $\mathrm{ABC}$. In previous phase 2 trials of CDK4/6 inhibitors on HR-positive and HER2-negative metastatic breast cancer, treatment with single agent abemaciclib yielded a DCR of $66.7 \%$ and a median PFS of 5.9 months in a 
heavily pretreated population (median prior lines of therapy, 2 for endocrine agents, 1 for chemotherapy) whereas single agent palbociclib yielded a similar DCR of $63.8 \%$ and a median PFS of 6.5 months in a moderately pretreated population (69\% with 1 line of prior endocrine therapy, $28 \%$ with prior chemotherapy) [16, 24]. Within the limitations of cross-trial comparison, the anti-tumor activity of single agent dalpiciclib reported in this trial was comparable to abemaciclib and palbociclib in treatment of $\mathrm{ABC}$. In general, our results were encouraging considering that the efficacy data of dalpiciclib were derived from a heavily pretreated population with refractory disease and from single agent treatment. Given the established synergic effects of dalpiciclib with letrozole, anastrozole and fulvestrant in a phase Ib trial (NCT03481998, Hengrui data on file) and the purpose to further improve anti-tumor activities, dalpiciclib is currently under evaluation in combination with endocrine therapy for HR-positive and HER2-negative ABC in two phase 3 trials: one in combination with letrozole or anastrozole in the frontline setting (NCT03966898) and one in combination with fulvestrant in the later-line setting (NCT03927456).

In summary, dalpiciclib showed acceptable safety profile and dose-dependent plasma exposure in Chinese patients with $\mathrm{ABC}$. The recommended phase 2 dose was determined to be $150 \mathrm{mg}$ QD. Preliminary evidence of clinical activity of dalpiciclib was observed.

\section{Abbreviations \\ ABC: Advanced breast cancer; AE: Adverse event; ALT: Alanine aminotransferase; AST: Aspartate aminotransferase; Cl: Confidence interval; CCND: Cyclin D; CDK4/6: Cyclin-dependent kinase 4/6; CR: Complete response; DCR: Disease control rate; DLT: Dose-limiting toxicity; HER2: Human epidermal growth factor receptor 2; HR: Hormone receptor; MTD: Maximum tolerated dose; ORR: Objective response rate; PD: Progressive disease; PFS: Progression-free survival; PK: Pharmacokinetics; PR: Partial response; Rb: Retinoblastoma protein; SD: Stable disease; TEAE: Treatment-emergent adverse event; ULN: Upper limit of normal}

\section{Supplementary Information}

The online version contains supplementary material available at https://doi. org/10.1186/s40364-021-00271-2.

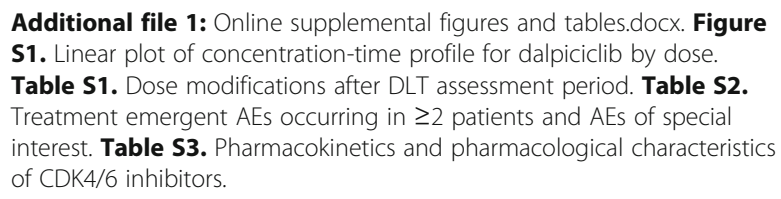

\section{Acknowledgements}

We thank the patients and their families for their participation in this study, as well as the study team at the National Cancer Center/Cancer Hospital of the Chinese Academy of Medical Sciences. We would also like to acknowledge Xiuzhi Wu, PhD (a medical writer at Hengrui) for medical writing support according to Good Publication Practice Guidelines.

\section{Authors' contributions}

Concept and design: BX, PZ, and JZ. Acquisition of data: BX, PZ, LG, WW, and MX. Data analysis: XZhang and GS. Interpretation of data: all authors. Drafting of the manuscript: BX, PZ, XZhang, GS, and XZhu. Critical revision of the manuscript for important intellectual content: all authors. Approval of the final manuscript: all authors.

\section{Funding}

The study was sponsored by Jiangsu Hengrui Medicine Co., Ltd.

Availability of data and materials

All data generated or analyzed during this study are included in this published article and its supplementary information files.

\section{Declarations}

Ethics approval and consent to participate

This trial was conducted in accordance with the principles of the Declaration of Helsinki and Good Clinical Practice guidelines. The study protocol was approved (reference \# 15-140/1067) by the ethics committee of the Cancer Hospital of the Chinese Academy of Medical Sciences (Beijing, China) and regulatory authorities. All patients provided written informed consent before enrollment.

\section{Consent for publication}

Not applicable.

\section{Competing interests}

BX has received research funding from Jiangsu Hengrui Medicine, served as an advisor or consultant for Novartis and Roche and served on Speaker's Bureaus for AstraZeneca, Eisai, Pfizer and Roche. XZhang, GS, XZhu and JZ are employees of Jiangsu Hengrui Medicine. Other authors declared no conflict of interests.

\section{Author details}

${ }^{1}$ National Cancer Center/Cancer Hospital, Chinese Academy of Medical Sciences and Peking Union Medical College, Beijing, China. ${ }^{2}$ Jiangsu Hengrui Medicine Co. Ltd, Shanghai, China.

Received: 14 December 2020 Accepted: 19 February 2021

Published online: 12 April 2021

\section{References}

1. Hanahan D, Weinberg RA. Hallmarks of cancer: the next generation. Cell. 2011;144(5):646-74.

2. O'Leary B, Finn RS, Turner NC. Treating cancer with selective CDK4/6 inhibitors. Nat Rev Clin Oncol. 2016;13(7):417-30.

3. Malumbres M, Barbacid M. Cell cycle, CDKs and cancer: a changing paradigm. Nat Rev Cancer. 2009;9(3):153-66.

4. Dean JL, Thangavel C, McClendon AK, Reed CA, Knudsen ES. Therapeutic CDK4/6 inhibition in breast cancer: key mechanisms of response and failure. Oncogene. 2010;29(28):4018-32.

5. Osborne CK, Schiff R. Mechanisms of endocrine resistance in breast cancer. Annu Rev Med. 2011;62:233-47.

6. Bosco EE, Knudsen ES. RB in breast cancer: at the crossroads of tumorigenesis and treatment. Cell Cycle. 2007;6(6):667-71.

7. Turner NC, Slamon DJ, Ro J, Bondarenko I, Im SA, Masuda N, et al. Overall survival with Palbociclib and Fulvestrant in advanced breast Cancer. N Engl J Med. 2018:379(20):1926-36.

8. Slamon DJ, Neven P, Chia S, Fasching PA, De Laurentiis M, Im SA, et al. Phase III randomized study of Ribociclib and Fulvestrant in hormone receptor-positive, human epidermal growth factor receptor 2-negative advanced breast Cancer: MONALEESA-3. J Clin Oncol. 2018;36(24):2465-72.

9. Sledge GW Jr, Toi M, Neven P, Sohn J, Inoue K, Pivot X, et al. The effect of Abemaciclib plus Fulvestrant on overall survival in hormone receptorpositive, ERBB2-negative breast Cancer that progressed on endocrine therapy-MONARCH 2: a randomized clinical trial. JAMA Oncol. 2020;6(1): $116-24$

10. Cristofanilli M, Turner NC, Bondarenko I, Ro J, Im SA, Masuda N, et al. Fulvestrant plus palbociclib versus fulvestrant plus placebo for treatment of hormone-receptor-positive, HER2-negative metastatic breast cancer that 
progressed on previous endocrine therapy (PALOMA-3): final analysis of the multicentre, double-blind, phase 3 randomised controlled trial. Lancet Oncol. 2016;17(4):425-39.

11. Sledge GW Jr, Toi M, Neven P, Sohn J, Inoue K, Pivot X, et al. MONARCH 2: Abemaciclib in combination with Fulvestrant in women with HR+/HER2advanced breast Cancer who had progressed while receiving endocrine therapy. J Clin Oncol. 2017;35(25):2875-84.

12. Finn RS, Martin M, Rugo HS, Jones S, Im S-A, Gelmon $K$, et al. Palbociclib and Letrozole in advanced breast Cancer. N Engl J Med. 2016;375(20):1925-36.

13. Hortobagyi GN, Stemmer SM, Burris HA, Yap YS, Sonke GS, Paluch-Shimon S, et al. Ribociclib as first-line therapy for HR-positive, advanced breast Cancer. N Engl J Med. 2016;375(18):1738-48.

14. Tripathy D, Im SA, Colleoni M, Franke F, Bardia A, Harbeck N, et al. Ribociclib plus endocrine therapy for premenopausal women with hormone-receptorpositive, advanced breast cancer (MONALEESA-7): a randomised phase 3 trial. Lancet Oncol. 2018;19(7):904-15.

15. Goetz MP, Toi M, Campone M, Sohn J, Paluch-Shimon S, Huober J, et al. MONARCH 3: Abemaciclib as initial therapy for advanced breast Cancer. J Clin Oncol. 2017;35(32):3638-46.

16. Dickler MN, Tolaney SM, Rugo HS, Cortés J, Diéras V, Patt D, et al. MONARCH 1, a phase II study of Abemaciclib, a CDK4 and CDK6 inhibitor, as a single agent, in patients with refractory HR(+)/HER2(-) metastatic breast Cancer. Clin Cancer Res. 2017;23(17):5218-24.

17. Wang J, Li Q, Yuan J, Wang J, Chen Z, Liu Z, et al. CDK4/6 inhibitor-SHR6390 exerts potent antitumor activity in esophageal squamous cell carcinoma by inhibiting phosphorylated Rb and inducing G1 cell cycle arrest. J Transl Med. 2017;15(1):127.

18. Long F, He Y, Fu H, Li Y, Bao X, Wang Q, et al. Preclinical characterization of SHR6390, a novel CDK 4/6 inhibitor, in vitro and in human tumor xenograft models. Cancer Sci. 2019;110(4):1420-30.

19. Eisenhauer EA, Therasse P, Bogaerts J, Schwartz LH, Sargent D, Ford R, et al. New response evaluation criteria in solid tumours: revised RECIST guideline (version 1.1). Eur J Cancer. 2009;45(2):228-47.

20. Bellet M, Ahmad F, Villanueva R, Valdivia C, Palomino-Doza J, Ruiz A, et al. Palbociclib and ribociclib in breast cancer: consensus workshop on the management of concomitant medication. Ther Adv Med Oncol. 2019;11: 1758835919833867.

21. Schwartz GK, LoRusso PM, Dickson MA, Randolph SS, Shaik MN, Wilner KD, et al. Phase I study of PD 0332991, a cyclin-dependent kinase inhibitor, administered in 3-week cycles (schedule 2/1). Br J Cancer. 2011;104(12):1862-8.

22. Infante JR, Cassier PA, Gerecitano JF, Witteveen PO, Chugh R, Ribrag V, et al. A phase I study of the Cyclin-dependent kinase $4 / 6$ inhibitor Ribociclib (LEE011) in patients with advanced solid tumors and lymphomas. Clin Cancer Res. 2016;22(23):5696-705.

23. DeMichele A, Clark AS, Tan KS, Heitjan DF, Gramlich K, Gallagher M, et al. CDK 4/6 inhibitor palbociclib (PD0332991) in Rb+ advanced breast cancer: phase II activity, safety, and predictive biomarker assessment. Clin Cancer Res. 2015;21(5):995-1001.

24. Malorni L, Curigliano G, Minisini AM, Cinieri S, Tondini CA, D'Hollander K, et al. Palbociclib as single agent or in combination with the endocrine therapy received before disease progression for estrogen receptor-positive, HER2negative metastatic breast cancer: TREnd trial. Ann Oncol. 2018;29(8):1748-54

25. Asghar U, Witkiewicz AK, Turner NC, Knudsen ES. The history and future of targeting cyclin-dependent kinases in cancer therapy. Nat Rev Drug Discov. 2015;14(2):130-46.

26. Groenland SL, Martínez-Chávez A, van Dongen MGJ, Beijnen JH, Schinkel AH, Huitema ADR, et al. Clinical pharmacokinetics and pharmacodynamics of the Cyclin-dependent kinase 4 and 6 inhibitors Palbociclib, Ribociclib, and Abemaciclib. Clin Pharmacokinet. 2020:59(12):1501-20.

27. Fogli S, Del Re M, Curigliano G, van Schaik RH, Lancellotti P, Danesi R. Drugdrug interactions in breast cancer patients treated with CDK4/6 inhibitors. Cancer Treat Rev. 2019;74:21-8.

28. US Food and Drug Administration: FDA warns about rare but severe lung inflammation with Ibrance, Kisqali, and Verzenio for breast cancer. 2019. https://www.fda.gov/drugs/drug-safety-and-availability/fda-warns-about-ra re-severe-lung-inflammation-ibrance-kisqali-and-verzenio-breast-cancer. Accessed 02 Feb, 2021

\section{Publisher's Note}

Springer Nature remains neutral with regard to jurisdictional claims in published maps and institutional affiliations.

\section{Ready to submit your research? Choose BMC and benefit from:}

- fast, convenient online submission

- thorough peer review by experienced researchers in your field

- rapid publication on acceptance

- support for research data, including large and complex data types

- gold Open Access which fosters wider collaboration and increased citations

- maximum visibility for your research: over $100 \mathrm{M}$ website views per year

At BMC, research is always in progress.

Learn more biomedcentral.com/submissions 